\title{
Design and Implementation of Multi-function Testing System Based on LXI Bus
}

\author{
Jiangtao Wei, Aijun Huang, Yong Zhou \\ Satellite Maritime Tracking and Controlling Department of China, Jiangyin 214431, P.R.China \\ weijtao@sina.com
}

Keywords: instrument bus; LAN Extensions for instrument; IVI driver; instrument interchangeable

\begin{abstract}
Based on LAN bus technology LXI instrumentation bus is a new generation after GPIB, VXI, PXI instrument bus, and it is also applicable for automation test system in the field of test and measurement. With the rapid development and popularity of the LXI instrument, more and more LXI instruments are used in the field of test and measurement and data analysis process, such as oscilloscope, signal source, digital multi-meter. As each instrument has its own software interface, it needs to switch between different software interfaces frequently in the course of the actual operation. To simplify the switching work, based on LXI bus we developed IVI interchangeable multifunctional testing system integrated instruments often used in our work. It makes full use of the program design technology, instrument technology and object oriented, and it combines the computer's capacity of processing and storage into one set. Practices prove that this system works well with easy operation and meets the requirements of design.
\end{abstract}

\section{Introduction}

With the development of Ethernet and standard PC technology, the technology of traditional GPIB can not meet the requirements of the modern testing industry. LXI (LAN Extensions for Instrument), which is the extension of local network in the field of instrument, is the new generation of test bus technology based on LAN and modular instrument platform.

LXI instruments are characterized by small volume, compact structure, no need of buttons, knobs or a conventional display, plug and play feature of virtual instrument system, etc. They also have the advantages of desktop instruments such as variety, easy operation and high performance. LXI adopts chassis unit structure conforming to IEC60297 standard which has high-speed I/O and reliable measurement capability. By using LAN technologies it facilitates to constitute local or regional measurement and control system, which can meet the demands for measurement and control system in the fields such as aerospace, weapon equipment, national defense security, information and communication, industrial manufacturing, transportation, energy production, medical equipment, agricultural and forestry production.

Based on the advantages of LXI, the LXI multi-function system integrates a variety of instruments so that the users can access the spectrum analyzer, digital multimeter, digital oscilloscopes and signal sources conveniently. By adapting C/S architecture it is very convenient for system expansion.

\section{Basic Concepts}

LXI Bus. LXI alliance released version 1.0 of the LXI standard in September 23, 2005. LXI is the achievement of the combination of measurement technology and network technology. As a new generation of test bus standard, it avoids the traditional architecture of complex and inefficient control mode. It can work with faster speed and easier connection assisting in the framework of IEEE1588 network synchronization standard. It is characterized with following features:

Open industry standards - - LAN and AC power supply is the most stable and longest life cycle standards in the open industry standards. And the manufacturers can transplant the existing instruments to the LXI bus platform easily because of the low cost of development. 
Backward compatibility - C Compared to VXI or PXI instrument, LXI bus instrument is smaller in volume. The LXI system can upgrade from the existing ATS (Automatic Test Systems) without re-configuration. The large card instrument (VXI, PXI) system is allowed to connect to LXI system. Figure 1 shows the hybrid bus test system based on LAN.

Interoperability - - The volume of the ATS system is reduced drastically with fewer types of modules. As a result the mobility and flexibility of the system are greatly improved, thus the user can constitute various test units for the target service efficiently.

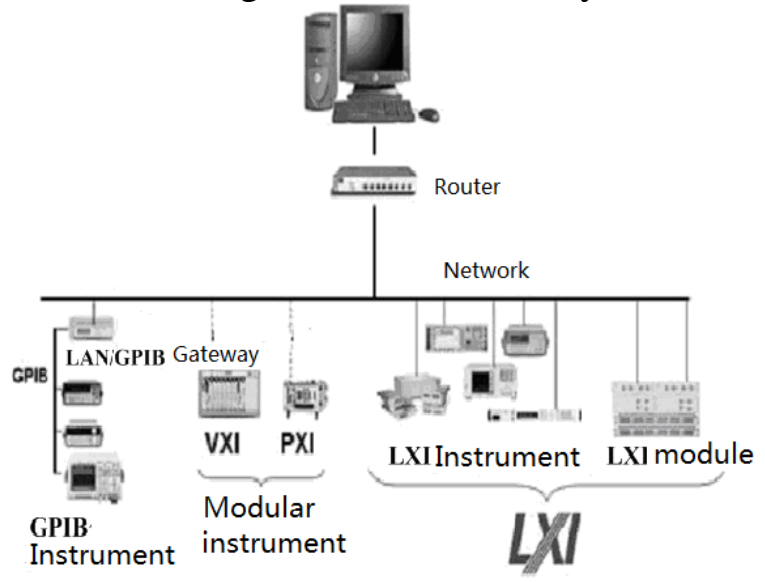

Fig. 1 LAN-based test systems

C/S Architecture. C/S structure is a typical two-layer architecture, which stands for Client/Server, namely client/server-side architecture. Its client contains one or more programs running on the user's computer. There are two types of server-side, one is the database server, the client-side access the server-side by connecting to a database. The other one is the Socket server, the server-side program communicates with the client-side by Socket. Due to the characteristics of LXI instruments, the Socket server is used in the design of this system.

IVI Driver Interface. The independence of the application and the driver is realized by IVI class driver and IVI configuration library. When the driver is changed it is unnecessary to change the application code correspondingly. IVI class driver provides a unified interface for the application and the specific instrument driver and the mapping relationship between the logical name and its related driver is stored in the IVI configuration library. When the instrument or the driver is changed, without modifying the application code, the only thing the users have to do is to reconfigure IVI configuration library.

According to API classification, IVI instrument drivers can be divided into IVI-C and IVI-COM, which are the production of the combination of COM and ANSI-C technology and virtual instrument.

As a universal development platform, VISA/IVI-COM (interactive virtual instruments) support module is very important. It is a universal instrument driver design standard, providing a common class library to guarantee the reliability and compatibility of the system. Additionally, it saves the time of development and simplifies the drive design. The basic structure is shown in Figure 2.

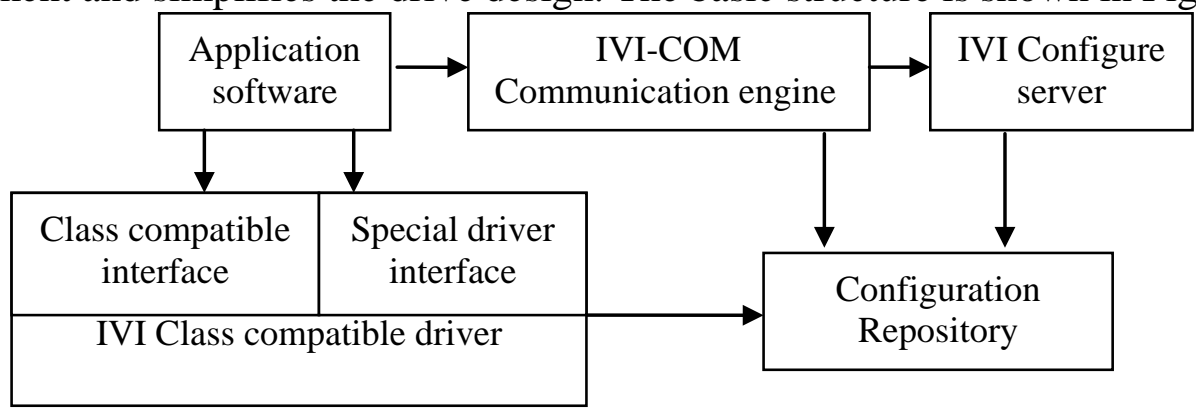

Fig. 2 Basic structure of IVI-COM 


\section{System Design}

Hardware Architecture. The hardware architecture of the whole test system is as follows:

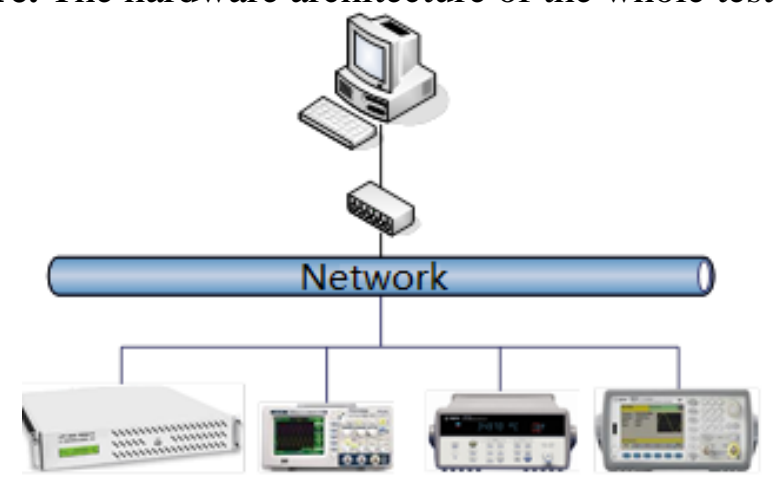

Fig. 3 Hardware architecture

The system is composed of main control computer, network/bus adapter, LXI instrument module. LXI instrument module in the system is directly connected with the computer via LAN hub. In order to enable the instrument's local or remote control, the standard Ethernet card configured by the host computer communicated to each instrument module in the system. To ensure the uniqueness and determinacy of the instrument identification, the static IP address is preferable used in the configuration of LXI system.

According to IVI interchangeability, any LXI instrument complied with the IVI specification can be interchanged to the instrument with same function, without modifying the software code.

System Configuration. In order to use IVI class drivers in the test program, the system must be configured firstly so that class drivers can interact with the specific instrument drivers. This step is accomplished by Configuration Server, which is in IVI Shared Components provided by IVI Foundation, and is composed of configuration store XML file and a COM object that can access, store XML file. The location of the instrument driver and the initialization configuration information are stored in configuration store XML file, including:

(1) All user-defined logical name is stored in the logical names folder. The logic name is used to identify the instrument. One can interchange the instrument the logical name referred to without changing the application code. The initialization information of drive properties such as State caching, simulation and so on, is also stored in this folder.

(2) The instrument drive folder contains information about where to find the specific drive information for each instrument in virtual instrument folder. In this folder, physical instrument address information is described in the form of VISA resources. The information of these folders is updated along with the system's increasing or reducing instruments. One can interchange the instruments by modifying the corresponding logical name of the specific instrument in the logical name folder.

The system configuration can be accomplished by the third-party software such as NI's MAX or Agilent IO. First configure the logical name, and then select the corresponding instrument drives and physical instrument to complete the configuration.

Software Mechanism. The computer is connected with each LXI instrument module via the switch. The LXI instrument driver module can be accessed by IVI software interface. The specified instrument modules can be accessed by instrument driver module. By accessory, the external signal can be connected conveniently, and the analog signals can be output to external instruments, thus various data analysis, signal simulation and other functions in industrial measurement fields can be realized.

Network communication module in this system is responsible for accurate data transmission between the main control computer and LXI instrument. The main control computer in the LAN communicates with the LXI instruments via TCP/IP protocol. After the LXI instrument is connected to the LAN, the computer in the network will control the LXI instrument remotely. To ensure reliable network data transmission, Connection-oriented Socket is used to communicate between the 
main control computer and LXI instrument. The parameters configuration and data transmitting and receiving of LXI instrument are also accomplished by Socket.

As it relates to multiple instrument modules, $\mathrm{C} / \mathrm{S}$ architecture is used to ensure the code reuse. The client is responsible for the work related to user interface, it sends the packaged data to the server. The server is responsible for receiving instructions from the client, and sending instructions to the instrument after parsing, and then returning the instrument results to the client. The software maintainability is ensured by the C/S architecture. When the new instrument is added to the system, the only thing to do is just add the corresponding client-side and a little modification to the server.

In the communication process, the client sends a request message to the server-side, the server-side transfers it to LXI instruments after parsing the control instructions. The server scans for the user request information regularly. It will continue to scan if there is no request, and if there is a client requests coming, the request information is queued according to the request time or priority. The highest priority access client is responded to, and then it waits for the next request. Then the responded data is returned to the client, and if there is no exception responded data, it will be displayed through the user interface real-timely, otherwise the appropriate error message will provide to the users after the data analysis and processing.

\section{Implementation Results}

Function of Software Panel. The multi-function test system software is developed by Studio Visual 2005. The software function of the LXI instrument is as follows:

\begin{tabular}{|l|l|}
\hline Spectrum analyzer & Spectrum monitoring, data analysis, data archiving and playback \\
\hline Signal source & $\begin{array}{l}\text { DC voltage measuring, AC voltage, DC current, AC current, four-wire } \\
\text { resistance, two-wire resistance, continuity, diode voltage }\end{array}$ \\
\hline Oscilloscope & $\begin{array}{l}\text { Waveform display, waveform adjustment, signal waveform real-time } \\
\text { display, measuring indicators commonly used }\end{array}$ \\
\hline Digital multimeters & Output sine, square, triangle, ramp, DC and arbitrary waveforms \\
\hline
\end{tabular}

Software panel. One can do the following work in the main software panel such as parameter setting, status display, spectrum monitoring, data analysis, data archiving and playback in the panel. Firstly, the configuration parameters, including the center frequency, bandwidth, should be set. And then the main control computer sends the parameters to spectrum analyzer via the network interface. And then the spectrum analyzer sends the monitor result back to the main control computer. The real-time monitoring module draws the spectrum after receiving the results, and the main characteristics of the spectrum, such as center frequency, bandwidth, resolution bandwidth, video bandwidth and reference level, are displayed in the panel.

Data recording and playback. The data can be recorded stored in a file, and users can store all the information in the current interface into a binary file. A frame head is added to the spectrum data. The frame head mainly includes data identification, recording time, recording length, center frequency, bandwidth, reference level, and so on.

The spectrum data playback module can reproduce the state of spectrum according to the recorded spectrum data. The time period of playback, the speed of playback can be selected manually in accordance with the log file for the spectrum data. It provides fast, normal and slow speed for users to choose. The query and analysis to critical spectrum data afterwards can be achieved by the playback.

\section{Summary}

Compared with GPIB, VXI and PXI bus, LXI bus has many advantages on the test system. In this paper, LXI is used to integrate various instruments. The C/S mode is adopted to make the system more convenient. 
Different from the traditional monitoring system, the LXI monitoring system has a significant character that the remote, real-time operation can be carried out through the transmission technology of the computer network, thus it effectively reduces the investment of human resource and financial in monitoring and testing work. The system can be used to set up the spectrum monitoring system of the test task, which is directly applied to monitor and analyze the critical waveforms.

\section{REFERENCES}

[1] LXI Consortium LXI Standard v1.1 2006.

[2] Yuanwen Cai, Xiaopeng Liang, Virtual Instrument Testing System Based on LXI Bus Technology [J]. Ordnance Industry Automation, 2010, 29(5).

[3] Ming Li, Na Gao, Key technology research of LXI-bus standard criterion[J], Electronic Measurement Technology, 2010, 33(8).

[4] Lin Wang, Xiang Huang, Design and Realization for Tele-measurement System Based on LXI Bus[J], Electrical Measurement \& Instrumentation, 2010, 47(5).

[5] Lingling Zheng, Gang $\mathrm{Xu}$, Remote control for spectrum analyzer based on LXI bus[J]. Electronic Measurement Technology, 2012, 35(1).

[6] Wilson Yu, LXI Instrument Development Platform Based on an Open Embedded Operating System[J], Chinese Journal of Scientific Instrument2007, 28(5). 